\title{
Time dependent model of the interplanetary Lyman $\alpha$ glow: applications to the SWAN data
}

\author{
E. Quémerais ${ }^{1,5}$, V. Izmodenov ${ }^{2,3,4}$, D. Koutroumpa ${ }^{1}$, and Y. Malama ${ }^{3,4}$ \\ 1 Service d'Aéronomie, IPSL, Université Versailles-Saint-Quentin, 91370 Verrières-le-Buisson, France \\ e-mail: eric.quemerais@aerov.jussieu.fr \\ ${ }^{2}$ Lomonosov Moscow State University, Department of Mechanics and Mathematics \& Institute of Mechanics, Moscow, Russia \\ Space Research Institute (IKI) Russian Academy of Sciences, Russia \\ 4 Institute for Problems in Mechanics Russian Academy of Sciences, Russia \\ 5 Institut d'Astrophysique Spatiale, Université Paris XI, 91400 Orsay, France
}

Received 2 July 2007 / Accepted 19 May 2008

\section{ABSTRACT}

\begin{abstract}
Aims. Previous results of the study of interplanetary Lyman $\alpha$ background data obtained by the SWAN-SOHO between 1996 and 2005 clearly show that the solar cycle variations of the solar parameters deeply affect the interplanetary background emission. In this work, we compare these observational results with a time-dependent modeling of the interplanetary background. The hydrogen distributions in the model are one-year averages.

Methods. The solar wind input in the model is derived from the omniweb dataset. The solar Lyman $\alpha$ flux values used to compute the radiation pressure are derived from the dataset of the SOLSTICE instrument. The hydrogen photo-ionization rate is extrapolated from the solar UV flux. These inputs are used to compute the hydrogen distribution in the heliosphere for two solar cycles. The resulting yearly averages of the interplanetary $\mathrm{H}$ distribution are then used as input for a radiative transfer model, which allows us to compute interplanetary background intensities, lineshifts, and linewidths for the geometries of the observations.

Results. We find that the upwind intensities computed from the model do not follow variations observed by SWAN-SOHO between 1996 and 2005. On the other hand, the lineshift variations during the solar cycle are correctly reproduced. Comparison of observed linewidths with model results show that we can reproduce the general trend of the linewidth data. Time-dependent variations are not fully reproduced.

Conclusions. The agreements obtained with the lineshifts and linewidths suggest that the velocity distribution of hydrogen is adequately represented by the model. On the other hand, we find that the temporal variations of the brightness data are not well reproduced by the model. To explain this, we suggest that the interface effects and radiation pressure are correctly represented whereas the ionization rate used as input in the model needs to be corrected. Further studies including anisotropy of the solar wind will be necessary to check this result.
\end{abstract}

Key words. interplanetary medium - ultraviolet: solar system

\section{Introduction}

The Solar Wind Anisotropy (SWAN) instrument on board the Solar and Heliospheric Observatory (SOHO) mission is dedicated to the study of the Lyman $\alpha$ interplanetary glow (Bertaux et al. 1995). This emission was first observed by two instruments on the OGO-5 mission (Bertaux \& Blamont 1971; Thomas \& Krassa 1971). It is caused by resonance scattering of the solar H Lyman $\alpha$ photons by neutral hydrogen atoms present in the interplanetary medium. The existence of hydrogen atoms in the inner heliosphere had been predicted before this first observation (Blum \& Fahr 1970). Following these initial studies, new experiments were dedicated to study this emission, first to characterize the hydrogen distribution in the heliosphere and also to study the solar wind, which is the main source of ionization of the hydrogen atoms in the inner heliosphere. Reviews of past experiments can be found in Ajello et al. (1987) and Quémerais et al. (1994).

In the inner heliosphere, hydrogen atoms that come from the interstellar medium can either be ionized by charge exchange with solar wind protons or photo-ionized by solar EUV photons. In both cases, the ionization rate is proportional to the flux from the Sun and varies like the inverse of the square of the distance to the Sun. Ionization by impacts with solar wind electrons may also occur, but the rate of this third mechanism is significantly lower than the rates of the other ionization mechanisms (Ruciński et al. 1996). Thomas (1978) published the classical hot model which describes the hydrogen distribution in the heliosphere under the influence of ionization effects, solar gravitation, and radiation pressure. Following observations of the interplanetary glow by Kumar \& Broadfoot (1979) and Bertaux et al. (1985), which suggested that the ionization fluxes were not always isotropic, Lallement et al. (1985) modified the classical hot model to include a possible dependence of the ionization rate on heliographic latitude. These authors showed that anisotropic ionization fluxes from the Sun could strongly modify the interplanetary glow pattern. From these results came the idea that the Lyman $\alpha$ glow could be used to derive the heliographic dependence of the ionization fluxes which then could be used to determine the solar wind mass flux at all latitudes (Bertaux et al. 1995).

The SWAN instrument has been operating almost continuously since January 1996, i.e. more than 10 years. During that period, starting just before the solar minimum of cycle 23 , the intensity pattern has changed dramatically as predicted in 
previous studies. Quémerais et al. (2006a) published an analysis of the intensity maps recorded by SWAN during the last decade. The data were used to derive the total ionization rate at all heliographic latitudes as it was announced in the initial proposal of the instrument. This analysis, which covers almost a 11-year solar cycle, is limited by two facts. First, it is based upon a stationary model, and second, it neglects the effects induced by the heliospheric interface on the hydrogen distribution in the heliosphere.

The heliospheric interface is the region of space where the expanding solar wind encounters the interstellar plasma. The heliopause is the contact discontinuity that separates the two plasmas. Because of charge exchange with protons, interstellar hydrogen atoms are strongly coupled with the interface. Indeed, Izmodenov et al. (2001) showed that roughly two hydrogen atoms out of three exchange a charge in the interface region thus strongly affecting the neutral distribution far from the Sun. Interface models were initiated at the end of the 1980's (Baranov 1990) but it took a few years to have results of self-consistent models including the neutral component (Baranov \& Malama 1993).

The second aspect that we will consider here is timedependence. Classical hot models are stationary. The variation of the solar parameters is taken into account by varying the input parameters (Lallement et al. 1985; Pryor et al. 1992). However, this is not fully satisfying because some hydrogen atoms take tens of years to cross the heliosphere. This means that the timescale of the phenomenon is on the order of the change of the solar parameters. Early time-dependent models were developed by Ruciński \& Bzowski (1995). Recently, Izmodenov et al. (2005a, 2008) have developed a new non-stationary kineticgasdynamic model of the heliospheric interface that includes time-dependent effects connected with the 11-year solar cycle. Some alternative models of the time-dependent interface were produced by Zank \& Muller (2003) and Scherer \& Fahr (2003). They use the multi-fluid approach for the H atom component. Comparisons of the kinetic and multi-fluid approaches are shown in Alexashov \& Izmodenov (2005), Heerikhuisen et al. (2006), Mueller et al. (2008), for the stationary case, and in Izmodenov et al. (2005a), for non-stationary case.

The aim of this paper is to compare results of interplanetary glow computations using the results of the time-dependent model of the heliospheric interface by Izmodenov et al. (2005a, 2008) as inputs for the hydrogen distribution, with the measurements of the SWAN instrument. The first section of this paper summarizes the model assumptions and relevant results of the time-dependent heliospheric interface model. In particular, the input parameters will be detailed. The second section compares results of the model intensities, lineshifts, and linewidths with the SWAN data. In the last section, we discuss these results.

\section{Time dependent hydrogen distributions}

\subsection{Model assumptions and boundary conditions}

There are two necessary requirements to model the interstellar $\mathrm{H}$ atoms for the interpretation of backscattered Lyman-alpha data: a) the model needs to take properly into account local effects that influence the distribution of the interstellar $\mathrm{H}$ atoms within 10-15 AU from the Sun; b) the model should address the effects of the heliospheric interface. In this paper, we employ the time-dependent version of the kinetic-fluid model by Izmodenov et al. (2005a, 2008) that satisfies both of these requirements. This model takes into account interaction of the solar wind (SW) with local interstellar cloud (LIC) also called circum-heliospheric insterstellar medium (CHISM). The LIC is assumed to have two components: H atoms and charged component consisting of electrons, protons, and ions of helium. The solar wind consists of electrons, protons and alpha particles in the model. All charged components are described by the one-fluid approximation. Magnetic fields are ignored in this model. For a study of the effects of the interstellar magnetic field, see, for example, Izmodenov et al. (2005b). The mathematical formulation of the governing equations are given in Izmodenov et al. (2005a, 2008).

For the pristine interstellar medium, we adopt: $V_{\mathrm{LIC}}=$ $26.4 \mathrm{~km} \mathrm{~s}^{-1}$ and $T_{\text {LIC }}=6527 \mathrm{~K}$ (Witte 2004; Möbius et al. 2004; Gloeckler et al. 2004; Lallement 2004a,b), $n_{\mathrm{H}, \mathrm{LIC}}=0.18 \mathrm{~cm}^{-3}$, $n_{\mathrm{p}, \mathrm{LIC}}=0.06 \mathrm{~cm}^{-3}$ (for argumentation see, e.g., Izmodenov et al. $2003,2004)$. The outflow boundary conditions in the tail region are assumed to be soft boundary conditions. For details of the computations in the tail direction, see Izmodenov \& Alexashov (2003), Alexashov et al. (2004a).

The boundary conditions at 1 AU are adopted from OMNIWeb of the Goddard Space Physics Data Facilities at http://omniweb.gsfc.nasa.gov/. The OMNIWeb data were used to get the solar wind parameters for the time interval defined by $t=\left[t_{1}, t_{2}\right]$ (values in decimal year are $t_{1}=1984.55$ and $t_{2}=2006.55$ ). For $t<t_{1}$ and $t>t_{2}$, we extrapolate the parameters using a periodicity equal to 22 years. The gasdynamic calculations were performed over 300 of 22-year cycles, enough to get a periodic solution in the entire computational domain including heliotail. Details of the numerical procedure is described in Izmodenov et al. (2005, 2008). Details of the boundary conditions are given in Izmodenov et al. (2008) and are not repeated here. As described in Izmodenov et al. (2008), for the considered model it is convenient to employ the solar wind mass, momentum and energy fluxes as the boundary conditions at the Earth's orbit. These fluxes are shown in Figs. 1A-D.

At the same time, the model takes into account effects of photoionization, solar gravity, and radiation pressure, which are only important within 10 to $20 \mathrm{AU}$ from the Sun. The ratio of the solar radiation pressure to solar gravitation force, the function $\mu\left(V_{r}, r, t\right)=\left|F_{\text {rad }}\right| /\left|F_{\text {grav }}\right|$ depends generally on $V_{r}, r$ and $t$. We assume in the model that absorption of solar photons within $10 \mathrm{AU}$ is negligible, and assume that

$\mu\left(V_{r}, r, t\right)=\mu_{0}(t) \mu_{1}\left(V_{r}\right)$

where $\mu_{0}(t)$ is the time variation of the line center flux as deduced from measurements of the solar illuminating flux, $\mu_{1}\left(V_{r}\right)$ is the solar Lyman $\alpha$ line profile as a function of doppler shift in the solar rest frame and normalized to 1 at line center. We assume here that the shape of the solar Lyman $\alpha$ profile does not change significantly over the solar cycle time-scale. Functions $\mu_{1}\left(V_{r}\right), \mu_{0}(t)$, which were used in the calculations, are shown in Figs. 1E, F. The $\mu_{0}(t)$ function was computed using the composite file of solar Lyman $\alpha$ flux produced by Woods et al. (2005b). This file is regularly updated to include the latest data. The solar line profile was obtained by SUMER/SOHO in 1996. A comparison of various SUMER measurements during the solar cycle is shown by Lemaire et al. (2005). Emerich et al. (2005) have published a detailed analysis of the relation between the line center flux (which is responsible for radiation pressure) and the measured total line flux. They find a small solar cycle dependence that changes the radiation pressure coefficient by $\pm 5 \%$ over the whole solar cycle. This effect is small enough to be neglected here. 

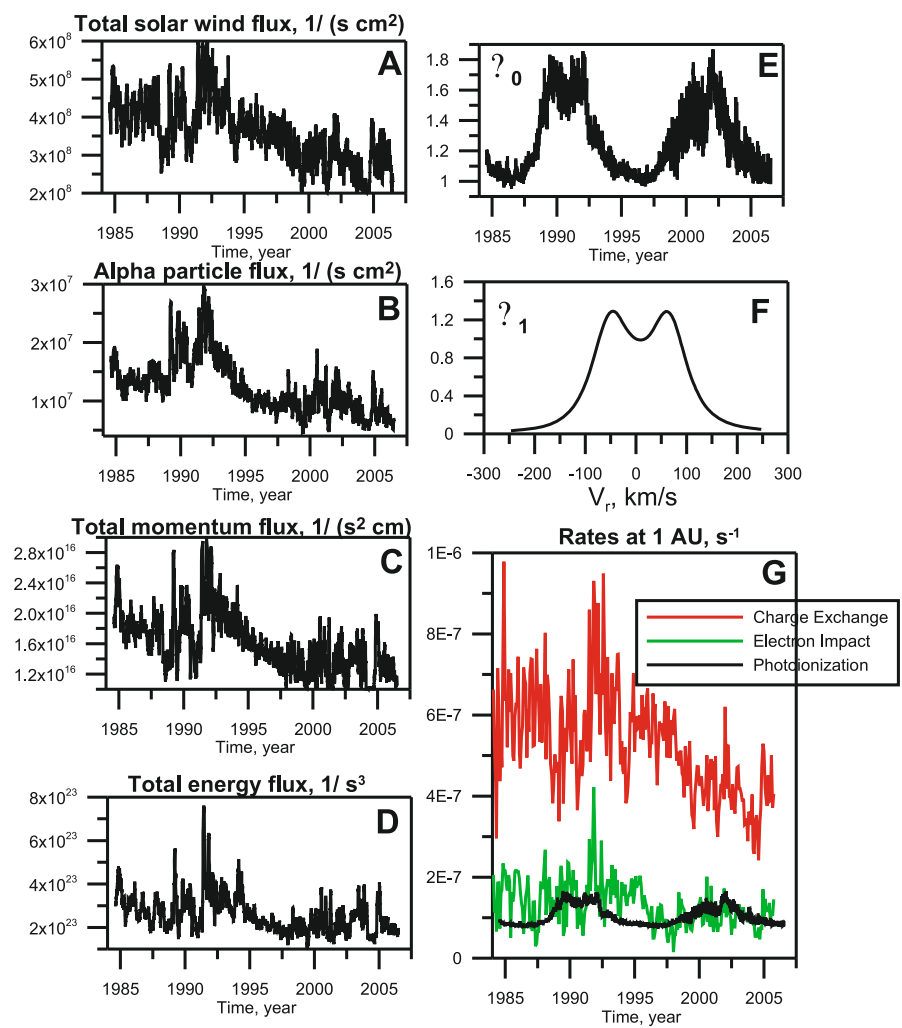

Fig. 1. We show 27-day averages of: A) the total solar wind flux; B) alpha particle flux; C) momentum flux; (D) energy flux; at the Earth's orbit as a function of time. E) $\mu_{0}$ is the ratio of the solar radiation pressure to solar gravitation acting on hydrogen atoms with $V_{r}=0$. F) $\mu_{1}$ is the Lyman $\alpha$ spectral line. G) Charge exchange (red curve), photoionization (black curve), and electron impact ionization (green curve) rates at the Earth's orbit as functions of time.

To get a self-consistent solution of such a system of governing kinetic-gasdynamic equations is not an easy task. Baranov et al. (1991) suggested the method of global iterations that allows for a stationary solution of the system of equations with stationary boundary conditions. Izmodenov et al. (2005a) have shown that the same method of global iterations works in the case of the non-stationary governing equations with the periodic boundary conditions. The solution of the system of governing equations was found under the reasonable assumption of the existence of a periodic solution. If such a solution would not exist then the global iteration scheme would not converge or the solution obtained would not satisfy the boundary conditions.

A more complex situation appeared when realistic solar cycle parameters were used. The solar wind data are available only for the three previous solar cycles and periodicity of the boundary conditions are not exactly established. In such a situation, it is important to understand how the solar wind conditions in the past influence the current plasma and $\mathrm{H}$ atom distributions. To answer this question, specific calculations with a so-called "broken" solar cycle were performed and their results were reported in Izmodenov et al. (2005a). In these calculations, it was assumed that the first of six solar cycles is broken'. The term "Broken" means that the solar wind parameter variations are not the same as in the "regular" solar cycle. We assumed that the solar wind dynamic pressure, which is the key parameter for the considered problem, is increased twofold as compared with the regular cycle. Such a significant and long lasting increase of the solar dynamic pressure has a significant impact on the interface structure. For example, the terminal shock (TS) of the

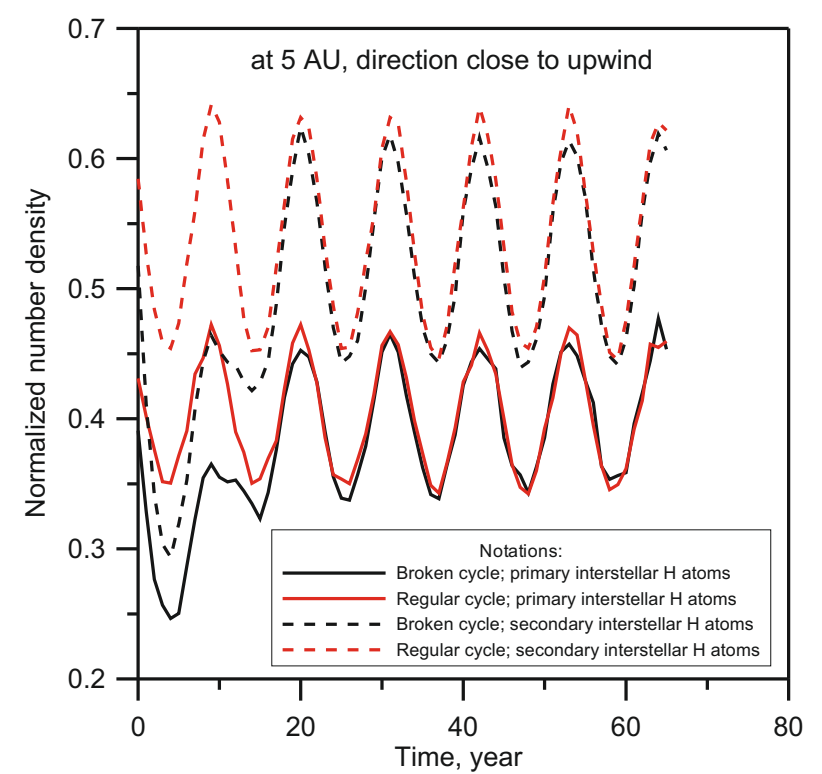

Fig. 2. Variations of number densities of primary (solid curves) and secondary (dashed curves) interstellar atoms for "ideal" (red curves) and "broken" solar cycle model calculations. Details of the models are given in Izmodenov et al. (2005a).

solar wind moves temporarily out by $\sim 10$ AU as compared with the regular or "ideal" solar cycle calculations, and the heliopause moves out by $\sim 5 \mathrm{AU}$. Nevertheless, the system returns back to its normal state rather rapidly. For example, the position of the TS moves back to its regular ideal solar cycle position in the middle of the following solar cycle. The page limitation of Izmodenov et al. (2005a) paper did not allow us to report how fast the interstellar atoms relax from the broken cycle to the regular cycle distributions. To know how fast this relaxation happens is a very important issue for the present work.

Figure 2 shows the number density of interstellar atoms at 5 AU for both regular and broken solar cycle calculations. It is seen that the broken cycle density relaxes to the regular cycle distribution in less than 11 years and with an accuracy of a few percents. This means that to interpret Lyman- $\alpha$ data from 1996 we should use the solar wind data starting from 1985 at least. In the calculations presented in this paper we adopted the solar wind data from 1984 to 2006 , which ensures the validity of the obtained $\mathrm{H}$ atom distributions starting from 1995.

The chosen numerical grid is sufficient for modeling both the global structure of the heliospheric interface and detailed distributions of $\mathrm{H}$ atoms inside the TS. The grid is not uniform and is adaptive to the discontinuities through a shock fitting procedure. The description and discussion of the spatial grid is given in Izmodenov et al. (2008). The time-resolution of the grid is also sufficient for the purposes of this paper. The time step in the numerical solution of the Euler equations was chosen as the minimum of two values. The first one is the time resolution of the inner boundary conditions at the Earth's orbit, which is typically daily or monthly averages as discussed in Izmodenov et al. (2008). The second one is the time step that follows from the numerical stability condition. The output plasma data are usually collected with the time resolution of the inner boundary conditions, i.e., daily or monthly averages. In principle, such high-time resolution plasma data can be employed by the MonteCarlo code. However, we choose one year averaging for $\mathrm{H}$ atom results. Firstly, the mean free path of the $\mathrm{H}$ atoms is large, and, therefore, the typical time for the charge exchange process is 


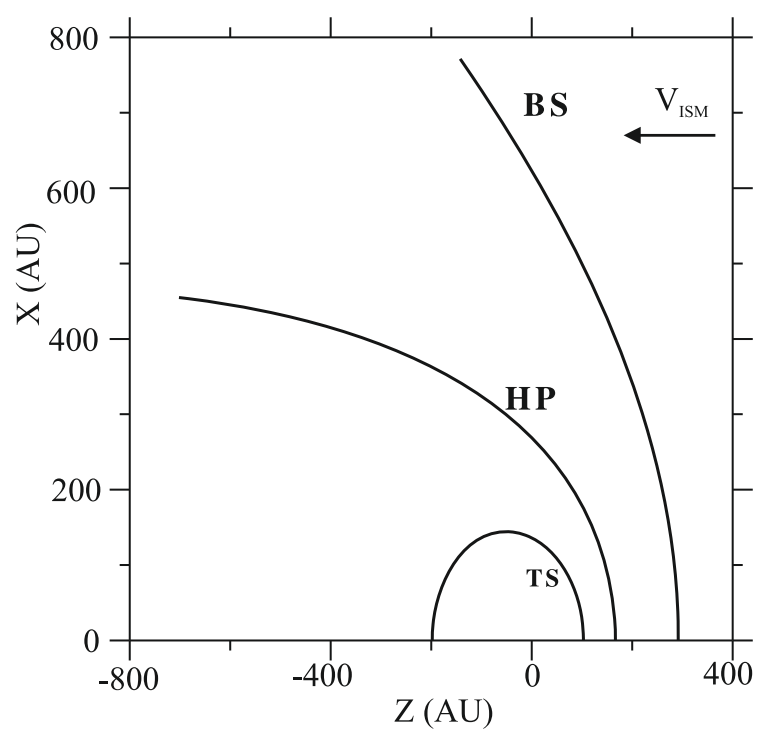

Fig. 3. The schematic structure of the heliospheric interface. The heliopause (HP) separates the solar wind from the interstellar plasma. The solar wind is decelerated from supersonic regime to subsonic at the termination shock (TS). The bow shock (BS) is formed in the supersonic interstellar plasma.

large too. Our estimations show that the characteristic time of charge exchange is larger than 1 year everywhere in our computational domain. Secondly, in this paper, the results of the theoretical calculations will be compared with one-year averaged backscattered Lyman- $\alpha$ data. With these two requirements, the calculations presented here were made with plasma data averaged over a one-year period. Note, also, that the sufficiency of the one-year averaged values was tested by comparing the computations with the time-resolution increased by a factor of 2 (i.e. by making 6-month averaged calculations). The results of two computations are identical.

Details of the results of the numerical solution describing the fluctuations of the termination shock, the heliopause, and the bow shock with the solar cycle and comparison of the velocity profiles with Voyager 2 data are given in Izmodenov et al. (2008). For the purposes of this paper, we focus on distributions of interstellar hydrogen inside $20 \mathrm{AU}$ and in the upwind direction.

\subsection{Results}

The general scenario of the solar wind interaction with the twocomponent interstellar medium was established by Baranov \& Malama (1993). The solar wind meets the interstellar charged component at the heliopause (HP, Fig. 3) which is the contact discontinuity separating the two media. Since both the solar wind and interstellar gas are supersonic flows, two shocks the termination shock (TS) and bow shock (BS) - are formed. Interstellar hydrogen atoms penetrate inside the plasma interaction region - the heliospheric interface - due to their large mean free path. Inside the heliospheric interface, the $\mathrm{H}$ atoms interact with decelerated and heated protons in the vicinity of the heliopause. As a result of the charge exchange, a new secondary hydrogen component is created. These secondary atoms reflect properties of the interstellar protons in the region between the HP and BS, i.e., they are hotter and slower as compared with original (or primary) interstellar atoms. Note that the secondary interstellar atoms are those that collectively make up the hydrogen wall around the heliopause. The hydrogen wall was initially predicted by Baranov et al. (1991) and then observed by Linsky \& Wood (1996) toward alpha-Cen. Now the heliospheric hydrogen wall is observed towards many directions (see, e.g., recent papers by Wood et al. 2007b and references therein).

Figure 4 presents the distributions of number densities, radial velocities, and temperatures of the primary and secondary interstellar components inside 20-50 AU for the upwind (Figs. 4A-C), crosswind (Figs. 4D-F), and downwind (Figs. 4G-I) directions. The distributions are shown for the period from 1996 to 2002 that is relevant to SWAN/SOHO observations. This period corresponds to the transition from solar minimum to solar maximum. The solar radiation pressure (and $\mu$ value) increases in this period (Fig. 1E). The increase of the repulsive force results in larger deviation of atom velocity and in corresponding decrease of radial velocities at small (inside 1015 AU for upwind) heliocentric distances. This effect is clearly seen (Fig. 4B) in upwind for both primary and secondary populations of interstellar atoms. Because of the increase of $\mu$ and the corresponding deflection of atom trajectories, one can expect a decrease of the number density of interstellar atoms from 1996 to 2003. This effect, however, is not observed (Fig. 4A). On the contrary, the number densities of both populations increase from 1996 to 2002. This is due to the prevailing effect of reduction of ionization by charge exchange during this period (Fig. 1G). The "kinetic" temperature does not change significantly from 1996 to 2002. A small increase of temperature for the secondary population is connected with the fact that, because of the increase in radiation pressure, a smaller fraction of the interstellar hydrogen atoms reach the space close to the Sun from the upwind direction, while a larger fraction comes from the sides. Similar effects are seen in the crosswind direction (Figs. 4D-F). The variations of radial velocity in the downwind region (Fig. $4 \mathrm{H}$ ) are smaller than for upwind and crosswind, while variations of densities are extended to larger distances up to 30-35 AU.

\section{Comparison with SWAN data}

The SWAN data used in this section have been presented by Quémerais et al. (2006a) and Quémerais et al. (2006b). The first work studies the intensity maps and derives monthly averages of total ionization rates of hydrogen as a function of heliographic latitude between 1996 and 2005. The second work studies line profiles reconstructed from $\mathrm{H}$ cell measurements and computes lineshifts and linewidths at different periods of the solar cycle.

Lineshifts can be translated in terms of velocities $(\Delta \lambda=$ $\lambda_{\mathrm{o}} v / c$ ). In that case, we call them line of sight (LOS) velocities. In the case of a monocinetic gas, the LOS velocity is close to the actual projected velocity of the gas (if one neglects radiative transfer effects). The models presented here are not monocinetic at all. In that case, the LOS velocity is the weighted mean of the distribution function of the emissivity of the gas on the line of sight multiplied by the extinction term. This is taken into account in the computation. However, we find it useful to express lineshifts in terms of dopplershift velocities.

In the same way, the linewidth can be expressed as a LOS temperature. There is a simple relation between the LOS temperature and the temperature of an isothermal and monocinetic (constant bulk velocity) gas distribution. Here, however, the complete modeling shows a much more complex case, which is fully taken into account in our computations. Still, linewidths will be expressed as LOS temperatures in Kelvin for the sake of simplicity. 

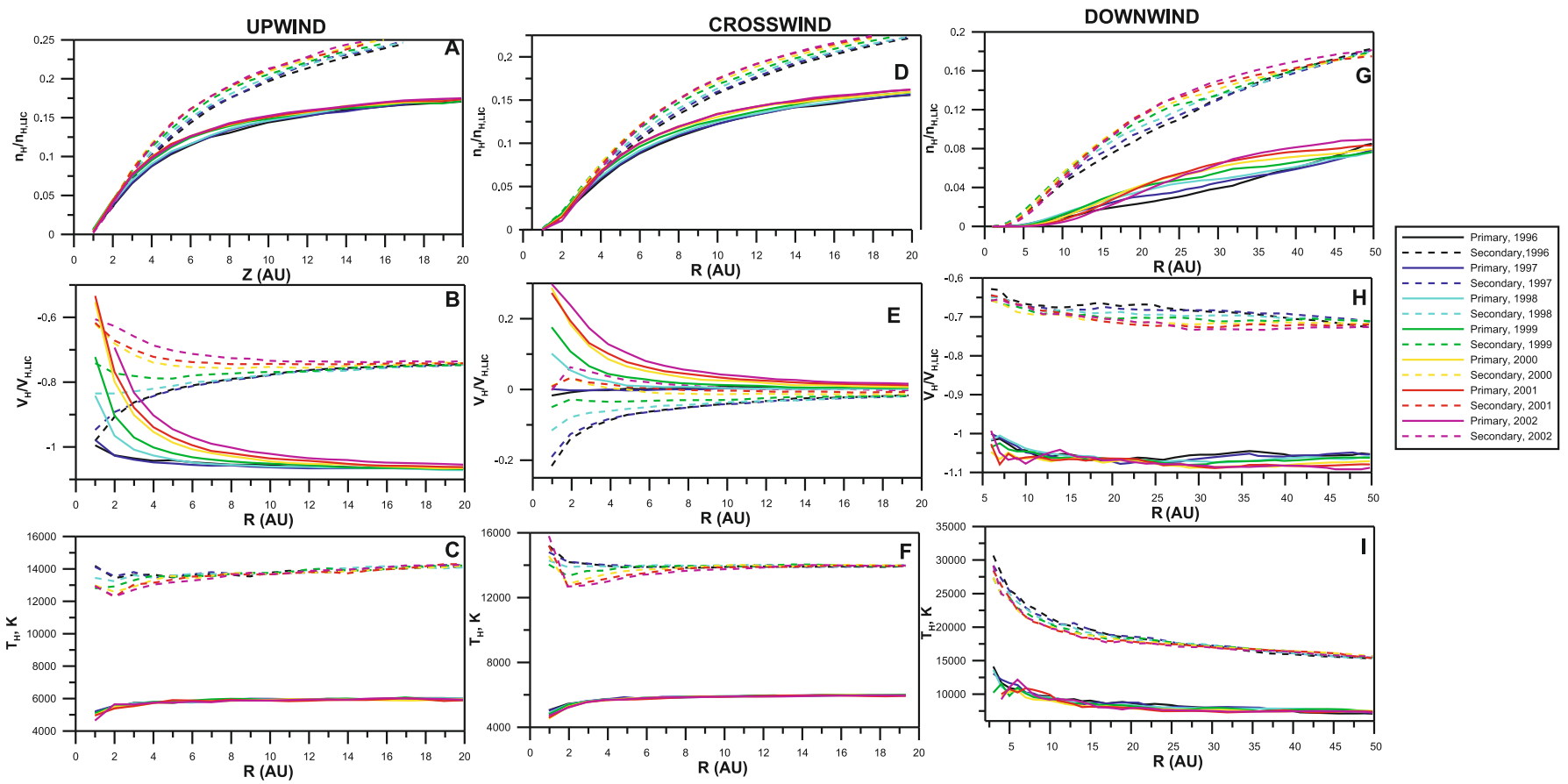

Fig. 4. One year averages of the number densities (A), D), G)), radial velocities (B), E), H)), and temperatures (C), F), I)) of primary (solid curves) and secondary (dashed lines) interstellar $\mathrm{H}$ atoms as function of the heliocentric distance for the upwind (right Col.), crosswind (middle Col.) and downwind (left Col.). Averaging are performed over 1 year from middle of June to middle of June of the following year. Label 1996 corresponds to period from June 1996 to June 1997.

\subsection{Intensities}

Quémerais et al. (2006a) have presented calibrated maps of background intensities measured by SWAN-SOHO. The relative calibration, i.e., the estimate of the sensitivity degradation as a function of time, was done by comparison of the count rates obtained on stars and by comparison of the two SWAN sensor units. The absolute calibration is based on the value published by Quémerais \& Bertaux (2002) obtained by comparison with a HST/GHRS measurement in 1996. Although this value is the official SWAN calibration factor, a comparison with a newer IP spectrum obtained by HST/STIS in March 2001 has raised a concern about the validity of the absolute calibration. In March 2001, it was estimated that the IP background intensity was equal to $1400 \mathrm{R}$ (John Clarke, private communication). Using the official SWAN calibration, we find a background intensity of $900 \mathrm{R}$ for the same date and geometry of observation. This suggests that the calibration factor should be increased by $55 \%$. In what follows, we will discuss results using the official calibration.

From the calibrated data set, we have selected a few maps when the observer is within $15^{\circ}$ of the upwind direction from the Sun, i.e., when the ecliptic longitude of SOHO is $252^{\circ} \pm 15^{\circ}$. From these maps, we have selected the observations for which the line of sight is within $15^{\circ}$ of the upwind direction. This was done to get observations as close to the radial case as possible. For each date of observation, we estimated the solar H Lyman $\alpha$ flux using the TIMED SEE data set available on the web (Woods et al. 2005b).

Figure 5 shows the data points (squares) extracted from the SWAN maps. The top curve (diamonds) shows the same data multiplied by a factor of 1.55 to show what the change in calibration would mean. The upwind intensity decreases after 2001, which suggests that the total hydrogen ionization rate is increasing as shown by Quémerais et al. (2006a). The intensity normalized to a constant flux (crosses) reaches a minimum in 2002, i.e.,

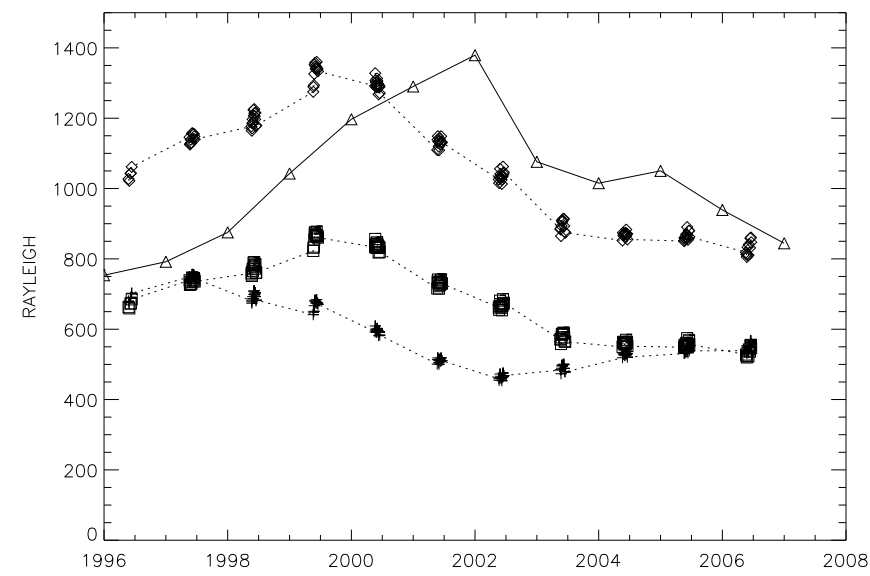

Fig. 5. IPH Intensity Data recorded within 10 deg of the upwind direction as a function of time. The observer's (SOHO) ecliptic longitude is equal to $252^{\circ} \pm 15^{\circ}$, i.e., is in the upwind direction from the Sun. The squares show the SWAN data. A second curve (diamonds) is shown based on a different absolute calibration (see text). The bottom curve (crosses) shows the data normalized to a constant solar flux.

more than one year after solar maximum. This suggests that the ionization cavity reaches its maximum size more than one year after the solar maximum, which is typically the kind of effect that can be described by a time dependent model.

The solid line in Fig. 5 shows the computed upwind radial intensity. For each yearly average we have computed the intensity, using the full radiative transfer code as presented in Quémerais \& Izmodenov (2002). The abscises is equal to the middle of the period of time during which the distributions are averaged. We have also derived the corresponding solar Lyman $\alpha$ flux at the 


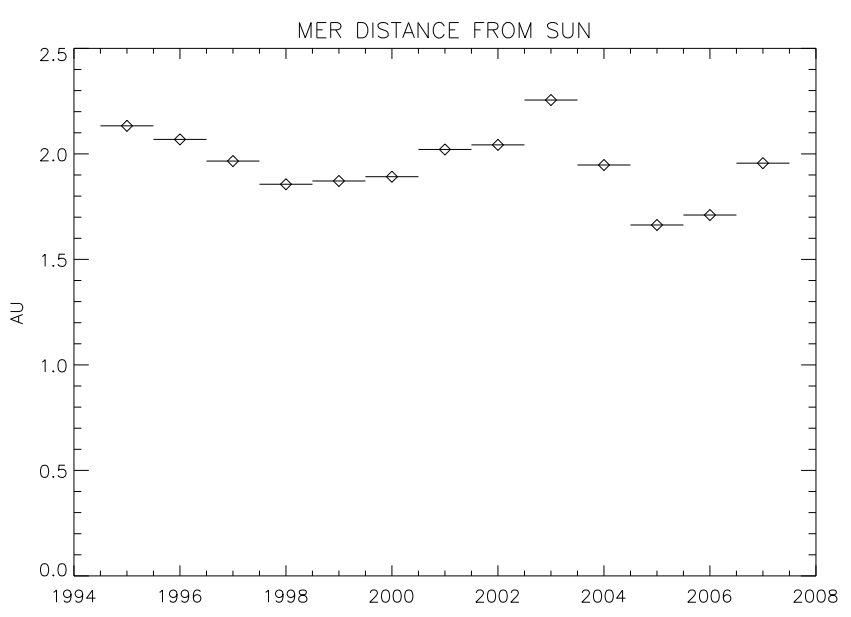

Fig. 6. Model computation of the distance between the MER in the upwind direction and the Sun. The MER position is mostly affected by radiation pressure. As radiatiation pressure increases, the MER is pushed back away from the Sun. There is a time delay of roughly two years between the radiation pressure minimum in 1996 and the minimum distance in 1998. The maximum distance is in 2003, i.e. two years after the radiation pressure maximum of 2001 . Values after 2003 are affected by the low-ionization rates used in the ionization input model.

same period and multiplied the model result by the corresponding flux. We see a strong discrepancy between data and model. The model values are constantly over the SWAN data (crosses) and the model peaks in 2002, while the peak value in the data is 2000-2001. This is a direct consequence of the input values used for the solar wind parameters and, therefore, for the charge exchange ionization rate. The omniweb solar wind data, which are used to determine the solar wind parameters (velocity and density), come from in-ecliptic measurements. Quémerais et al. (2006a) showed, see Fig. 14 of their paper, that the ionization rate derived from these values do not increase with the cycle. In fact, it decreases a little. As a consequence, data and model show different time variations. There is a shallow local minimum in the 2003-2004 model. It is due to the effect of radiation pressure which pushes hydrogen atoms away from the Sun. However, radiation pressure is not efficient enough to cause an increase of the size of the ionization cavity as the one that is observed from the data.

The model/data comparison is better if we multiply the SWAN data by a factor 1.55. The mean values are in better agreement, but the peak values are still out of phase. This result suggests that the SWAN calibration should be checked. Comparisons with SPICAM on Mars Express and SPICAV on Venus Express will be performed.

Figure 6 shows the distance between the Sun and the maximum emissivity region (MER) for each model. The MER is the point of the inner heliosphere, that has the largest Lyman $\alpha$ emissivity. The distance between the Sun and this point, which is located upwind from the Sun, is good indicator of the size of the cavity. As expected from the intensity model values, the MER is furthest away from the Sun in 2003 because of the effect of radiation pressure. From this figure, we also note that the cavity is smaller in 1998 than in 1996, which is certainly a time dependent effect in the model.

In conclusion, the upwind radial intensity computed from the model show that there is a strong disagreement between the SWAN intensity data and the solar wind parameters used as input to the time dependent model. The data suggest that the ionization cavity surrounding the Sun increases strongly in size, reaching a maximum extent in 2002-2003. Because the solar cycle increase of radiation pressure alone cannot explain this effect, this means that the total hydrogen ionization rate has to increase. Quémerais et al. (2006a) showed that the ionization rate derived from the SWAN data doubles between solar minimum and solar maximum. On the other hand, in-ecliptic solar wind measurements from various spacecraft and collected in the omniweb database can be used to estimate the time dependence of the ionization rate. The result does not show an increase after the solar minimum of 1996. In fact, there is a small decrease over the whole 1996 to 2005 period. This discrepancy will be discussed further.

\subsection{Lineshifts}

Quémerais et al. (2006b) used the SWAN H cell data to reconstruct the interplanetary line profile. The reconstruction method is described in Quémerais et al. (1999). From these profiles, we can compute lineshifts and linewidths. Although these values are more difficult to interpret than local distributions, they still provide information on the velocity distribution of the $\mathrm{H}$ atoms in the inner heliosphere.

From the SWAN H cell data, Quémerais et al. (2006b) found that the upwind lineshift in the solar rest frame, expressed in terms of the equivalent velocity (LOS velocity), changed from $25.7 \mathrm{~km} \mathrm{~s}^{-1}$ down to $21.4 \mathrm{~km} \mathrm{~s}^{-1}$ between the solar minimum in 1996 and the solar maximum in 2001. The low value of the lineshift at solar maximum was confirmed independently by an HST/STIS spectrum obtained in March 2001 (Clarke et al. 2007, private communication). The main factor for this change is certainly the change in radiation pressure with increasing solar activity. The numerical values given by Quémerais et al. (2006b) are shown in Table 1.

Figure 7 shows a comparison of the IP upwind lineshift derived from the time dependent model with the SWAN values. The variation between solar minimum and solar maximum found in the SWAN data is correctly represented by the model. At solar minimum, there is a small difference of $\sim 1 \mathrm{~km} \mathrm{~s}^{-1}$ between data and model.

A similar study was performed on a stationary model to see if the upwind lineshift variations could be reproduced simply by changing the solar parameters in a hot model. For this work, we used a refined hot model by combining two hydrogen populations to approximate the results of Izmodenov et al. (2001). The cold population bulk velocities and temperature, equal to $28 \mathrm{~km} \mathrm{~s}^{-1}$ and $6000 \mathrm{~K}$ respectively, are very close to the Helium parameters (Witte et al. 2004). The change in velocity from $26 \mathrm{~km} \mathrm{~s}^{-1}$, as derived for helium, up to $28 \mathrm{~km} \mathrm{~s}^{-1}$ is due to selection effects when crossing the interface region. The hot population, created after charge exchange, has a velocity of $18 \mathrm{~km} \mathrm{~s}^{-1}$ and a temperature of $16000 \mathrm{~K}$. Izmodenov et al. (2001) found that roughly 2 hydrogen atoms out of 3 crossing the interface region exchange their charge. Inside the heliosphere, the distributions are computed independently for the two populations because of the lack of collisions. Intensities and line profiles can be computed and then added. Corrections for radiative transfer effects as computed by Quémerais \& Izmodenov (2002) are also included.

Figure 8 shows how the upwind lineshift changes with radiation pressure in the case of this simplified model. The solid lines correspond to the sum of the two components. One is for a high-ionization rate case and the other for a low-ionization rate. From these computations, we find that lineshifts derived from stationary models require very large variations of the radiation 
Table 1. Upwind Lineshift for various orbits.

\begin{tabular}{lcccccc}
\hline \hline Year (starts in june) & 1996 & 1997 & 1999 & 2000 & 2001 & 2002 \\
\hline$V($ year $)\left(\mathrm{km} \mathrm{s}^{-1}\right)$ & $-25.7 \pm 0.2$ & $-25.3 \pm 0.2$ & $-22.5 \pm 0.5$ & $-21.5 \pm 1.2$ & $-21.5 \pm 0.3$ & $-21.4 \pm 0.5$ \\
\hline$V($ year $)-V(1996)\left(\mathrm{km} \mathrm{s}^{-1}\right)$ & 0. & 0.4 & 3.2 & 4.2 & 4.2 & 4.3 \\
\hline
\end{tabular}

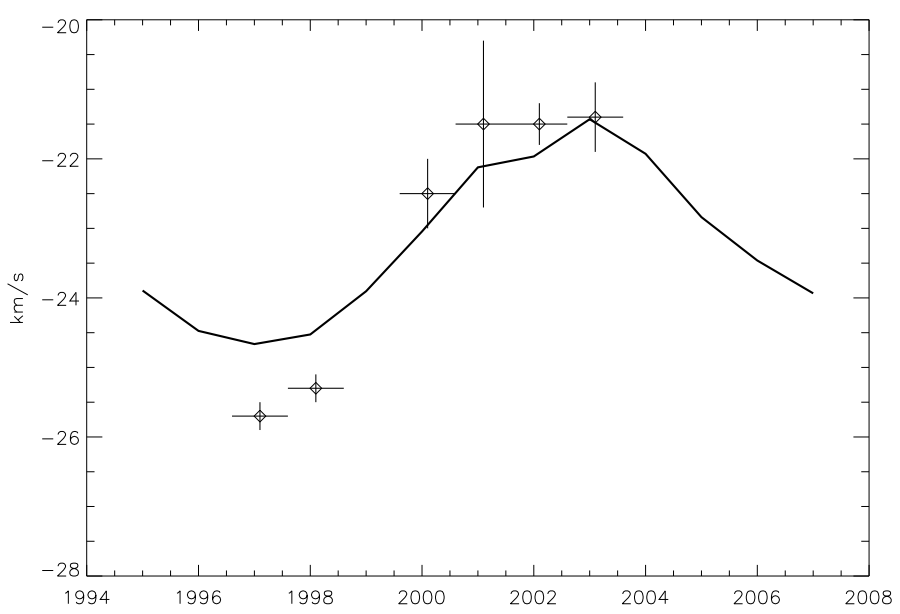

Fig. 7. Lineshifts (expressed in $\mathrm{km} \mathrm{s}^{-1}$ ) in the upwind direction as a function of time. The SWAN values from Quémerais et al. (2006b) are shown by the diamonds. Vertical bars show the uncertainty and horizontal bars show the time period of the data used to derive the value (one year). The thick line shows the full radiative transfer computation results. There is a correct agreement between data and model for the lineshifts.

pressure coefficient (noted $\mu$, it measures the ratio of radiation pressure over solar attraction force) to match the observed values of the IP lineshift. Solar Lyman $\alpha$ flux variations from Woods et al. (2005b) indicate that $\mu$ varies between 0.9 and 1.6 during the solar cycle. Such an amplitude of variation of the radiation pressure can cause a change in upwind lineshift around $2 \mathrm{~km} \mathrm{~s}^{-1}$. However, the observed variation is larger than $4 \mathrm{~km} \mathrm{~s}^{-1}$ (Table 1). This shows that, given the known range of the radiation pressure coefficient, stationary models are not able to reproduce the observed cycle variation of the IP H Lyman $\alpha$ shift while time dependent models can.

\subsection{Linewidths}

Quémerais et al. (2006b) have also studied the variation of the IP H Lyman $\alpha$ linewidth during the cycle. They considered radial (antisolar line of sight) directions from upwind to downwind. They found that the linewidth does not vary monotically from upwind to downwind. This was assumed to be caused by the shift between the cold and hot populations but was not modeled. In 1996 and 1997, they found a minimum around $60^{\circ}$ from upwind. As appears in Fig. 7 of Quémerais et al. (2006b), they also found that this minimimum is displaced in the period 20002002 around 30-40 deg from upwind and is not clearly apparent in 1999.

Figure 9 shows the antisolar linewidth as a function of the upwind angle for the 1997 model and the 2003 model. We should point out that a model labeled 1997 uses distributions averaged from mid-1997 to mid-1998. First, we find that the equivalent temperature values in the model are between $12000 \mathrm{~K}$ and $16000 \mathrm{~K}$ which corresponds well to the data. The 2003 model also shows a local minimum around $60 \mathrm{deg}$ from upwind. The

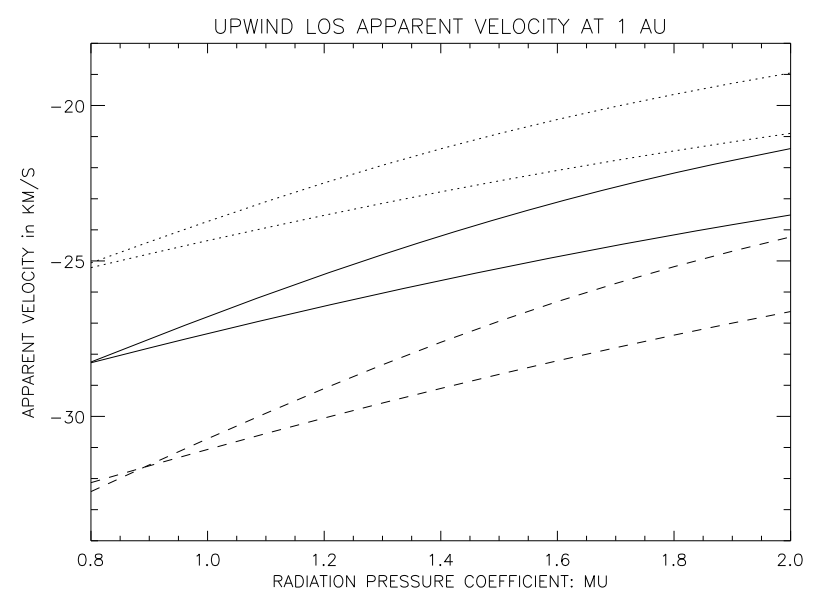

Fig. 8. Upwind lineshift derived from the two-population line profile computations. The abscisse is the radiation pressure coefficient. The thick lines are computed for an ionization rate of $12 \times 10^{-6} \mathrm{~s}^{-1}$ and the thin lines for a value of $6 \times 10^{-6} \mathrm{~s}^{-1}$. The two dotted lines correspond to the slow component, the dashed line to the fast component and the solid lines correspond to the sum.

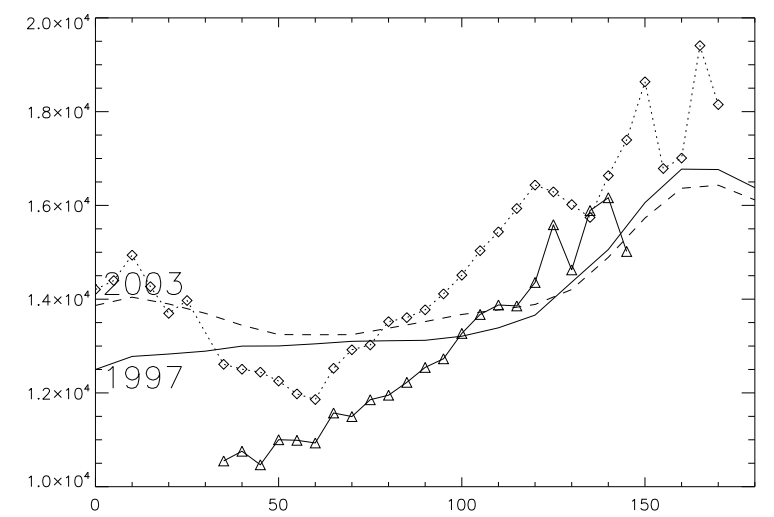

Fig. 9. Model computation of the linewidth as a function of the angle with upwind. The solid line shows the model in 1997 when the values are lowest. The dashed line shows the values obtained in 2003. The linewidth is noticably larger close to the upwind direction with an excess close to $2000 \mathrm{~K}$. In 2003 , there is a local minimum close to $60 \mathrm{deg}$ from upwind. For comparison, we have added two curves from Quémerais et al. (2006b), which show the values derived from the SWAN data in 1996-1997 (diamond+dotted line) and in 20012002 (triangle+dotted line). For the 2001 data, the values below $30^{\circ}$ and above $150^{\circ}$ are not available because of stellar contamination.

minimum in the model is more shallow (Fig. 9). It is also interesting to note that the 1997 model does not have a local minimum, which is also suggested by the 1999 and 2001 data curve from Quémerais et al. (2006b).

For comparison we have added two curves from Fig. 7 of Quémerais et al. (2006b) which are representative of solar minimum and solar maximum conditions. The diamond and solid line show the 1996-1997 data and the triangles and solid line 
show the 2001-2002 data. Because of stellar contamination in the data close to the galactic plane, data within $30^{\circ}$ of upwind and within $30^{\circ}$ of downwind have large uncertainties. The overall variation between upwind and downwind seem larger in the data. However this may be due to uncertainties in the measurements, which are shown in Quémerais et al. (2006b). The two data curves, solar minimum to solar maximum, also show larger differences than the two model curves. The existence of the local minimum at $60^{\circ}$ from upwind is shown in the 1996-1997 data but not in the 2001-2002 data. The solar minimum model curve shows a local minimum at $60^{\circ}$, which is not seen in the model curve obtained for solar maximum conditions. This is the reverse of what is seen in the data and suggests that some effect is not taken into account in the model. Overall, we find a correct agreement between the linewidths derived from the data and the results of the present work.

We also used our two-population stationary hot model to compute linewidths for radial lines of sight going from upwind to downwind. As in the previous section, we used two values of ionization rate to represent high-ionization rate conditions $\left(1.2 \times 10^{6} \mathrm{~s}^{-1}\right)$ and low-ionization rate $\left(0.6 \times 10^{6} \mathrm{~s}^{-1}\right)$. We could not reproduce the local minimum around $60 \mathrm{deg}$ from upwind in these models showing once again that a time dependent model is required here.

\section{Conclusion and discussion}

The comparison between observed and modeled IP Lyman $\alpha$ lineshifts and linewidths shows that the time dependent model gives a good representation of the hydrogen distribution in the inner heliosphere.

Lineshifts are mainly affected by radiation pressure effects. Thus, being able to reproduce the cycle variations of the lineshifts shows that radiation pressure is correctly taken into account in the model. There is still a small discrepancy between data and model but it may be an effect of non-isotropy of the ionization rate.

Linewidths are characterized by the existence of two populations, which causes a increasing of the width of the IP line. Furthermore, the existence of a local minimum of linewidth at $60^{\circ}$ from upwind is a strong sign of the existence of the two populations of hydrogen with the hot population being created at the heliospheric interface. A model with only one population cannot have this local minimum of the radial linewidth as a function of the angle from upwind.

On the other hand, our computations show that the observed and modeled intensities do not have the same temporal variations. The time-dependent model intensity variation is determined by the solar wind parameters, which are used as input in the time dependent models. These parameters are obtained from solar wind density and velocity values given by the omniweb database. These values come from local and in-ecliptic measurements. The SWAN intensity variation is determined from the data normalized to a constant solar flux. The solar Lyman $\alpha$ flux value is derived from TIMED SEE data (Woods et al. 2005b, latest file updated in September 2007). While the normalized model intensity shows a small increase between 1996 to 2005 , the SWAN data show a strong decrease after 1999, which suggests that the ionization rate increases a lot, by a factor of 2 according to Quémerais et al. (2006a).

Another problem comes from the fact that the SWAN data are constantly lower than the computed model values. This appears also in Quémerais et al. (2006a) where the derived ionization rates are larger than the values computed from in situ measurement data. This problem is alleviated if we use a new calibration factor obtained from a March 2001 IP background measurement made by STIS-HST.

According to our estimates using WIND solar wind electron data (Issautier et al. 2005), electron impact ionization cannot explain this discrepancy. The ionization rates computed from these data are at best one order of magnitude below the rates for charge exchange with solar wind protons. Similar results were obtained by Ruciński et al. (1996).

The other explanation is that the omniweb database give only a very limited view of the solar wind parameters at the solar maximum. Being local, these measurements sample only one type of solar wind and only in the ecliptic plane. On the other hand, the SWAN data are global and averaged over a month. It takes a few months for a hydrogen atom to cross the inner heliosphere and atom trajectories are not limited to the ecliptic plane. If this is the case, the SWAN data show that at solar maximum the solar wind conditions are not well represented by in-ecliptic measurements and that the solar wind mass flux or ram pressure are higher than what can be estimated from the omniweb database.

Acknowledgements. SOHO is a mission of international cooperation between ESA and NASA. SWAN was financed in France by CNES with support from CNRS and in Finland by TEKES and the Finnish Meteorological Institute.

The authors wish to thank International Space Science Institute in Bern (ISSI) for providing support for team meetings.

V. I. and Y. M are supported also in part by RFBR grant 07-02-01101-a.

Y. M. was partly supported by grant RFBR 06-02-72557-CNRS-L-a. V.I. was also supported by "Dynastia Foundation".

\section{References}

Ajello, J. M., Stewart, A. I., Thomas, G. E., \& Graps, A. 1987, ApJ, 317, 964 Ajello, J. M., Pryor, W. R., Barth, C. A., et al. 1994, A\&A 289, 283 Alexashov, D., \& Izmodenov, V. V. 2005, A\&A, 439, 1171

Alexashov, D. B., Izmodenov, V. V., \& Grzedzielski, S. 2004, Adv. Space Res., 34, 109

Baranov, V. B. 1990, Space Sci. Rev., 52, 89

Baranov, V. B., \& Malama, Yu. G. 1993, J. Geophys. Res., 98, 15157

Baranov, V. B., \& Izmodenov, V. V. 2006, Fluid Dyn., 41, 689

Baranov, V. B., Lebedev, M., \& Malama, Y. 1991, ApJ, 375, 347

Bertaux, J. L., Blamont, J. E., Mironova, E. N., et al. 1977, Nature, 270, 156

Bertaux, J. L., Lallement, R., Kurt, V. G., \& Mironova, E. N. 1985, A\&A, 150, 82

Bertaux, J. L., Kyrölä, E., Quémerais, E., et al. 1995, Sol. Phys., 162, 403

Bertaux, J. L., Quémerais, E., Lallement, R., et al. 1997, Sol. Phys., 175, 737

Blum, P. W., \& Fahr, H. J. 1970, A\&A, 4, 280

Emerich, C., Lemaire, P., Vial, J. C., et al. 2005, Icarus, 178, 429

Gloeckler, G., Moebius, E., \& Geiss, J. 2004, A\&A, 426, 845

Heerikhuisen, J., Florinski, V., \& Zank, G. P. 2006, J. Geophys. Res., 111, A06110

Issautier, K., Perche, C., Hoang, S., et al. 2005, Adv. Space Res., 35, 2141

Izmodenov, V., \& Alexashov, D. 2003, Astron. Lett., 29, 58

Izmodenov, V., \& Alexashov, D. 2006, AIP Conf. Proc., 858, 14

Izmodenov, V. V., Gruntman, M., \& Malama, Y. G. 2001, J. Geophys. Res., 106, 10681

Izmodenov, V., Malama, Y. G., \& Ruderman, M. S. 2005a, A\&A, 429, 1069

Izmodenov, V., Alexashov, D., \& Myasnikov, A. 2005b, A\&A, 437, L35

Izmodenov, V., Malama, Y. G., \& Ruderman, M. S. 2008, Adv. Space Res., 41, 318

Koutroumpa, D., Lallement, R., Bertaux, J. L., Quémerais, E., \& Ferron, S. 2005 In: Proceedings of the SOHO 16/ Solar Wind 11 Symp., ed. T. Zurbuchen, \& B. Fleck, ESA SP-592

Kumar, S., \& Broadfoot, A. L. 1979, ApJ, 228, 302

Lallement, R., Bertaux, J. L., \& Dalaudier, F. 1985, A\&A, 150, 21

Lallement, R., Raymond, J. C., Vallerga, J., et al. 2004a, A\&A, 426, 875

Lallement, R., Raymond, J. C., Bertaux, J. L., et al. 2004b, A\&A, 426, 867

Lallement, R., Quémerais, E., Bertaux, J. L., et al. 2005, Science, 307, 1447

Lemaire, P., Emerich, C., Vial, J. C., et al. 2005, Adv. Space Res., 35, 384

Linsky, J. L., \& Wood, B. E. 1996, ApJ, 463, 254

Moebius, E., Bzowski, M., Chalov, S., et al. 2004, A\&A, 426, 897

Opher, M., Stone, E. C., \& Liewer, P. C. 2006, ApJ, 640, L71

Opher, M., Stone, E. C., \& Gombosi, T. I. 2007, Science, 316, 875 
Maher, L. J., \& Tinsley, B. A. 1977, J. Geophys. Res., 82, 689

Müller, H.-R., Florinski, H., Heerikhuisen, J., et al. 2008, A\&A, in press [arXiv:0804.0125]

Pogorelov, N. V., \& Zank, G. P. 2006, ApJ, 636, L161

Pogorelov, N. V., Zank, G. P., \& Ogino, T. 2006, ApJ, 644, 1299

Pryor, W. R., Ajello, J. M., Barth, C. A., et al. 1992, ApJ, 394, 363

Quémerais, E. 2000, A\&A, 358, 353

Quémerais, E., \& Bertaux, J. L. 2002, ISSI Scientific Report SR-002, ed. A. Pauluhn, M. C. E. Huber, \& R. von Steiger. (Noordwijk, The Netherlands: ESA Publications Division), 203

Quémerais, E., \& Izmodenov, V. 2002, A\&A, 396, 269

Quémerais, E., Bertaux, J. L., Sandel, B. R., \& Lallement, R. 1994, A\&A, 290, 941

Quémerais, E., Bertaux, J. L., Lallement, R., et al. 1999, J. Geophys. Res., 104, 12585

Quémerais, E., Lallement, R., Ferron, S., et al. 2006a, J. Geophys. Res., 111, A09114
Quémerais, E., Lallement, R., Bertaux, J. L., et al. 2006b, A\&A, 455, 1135

Ruciński, D., Cummings, A. C., Gloeckler, G., et al. 1996, Space Sci. Rev., 78, 73

Ruciński, D., \& Bzowski, M. 1995, A\&A, 296, 248

Scherer, K., \& Fahr, H. J. 2003, Ann. Geophys., 21, 1303

Thomas, G. E., \& Krassa, R. F. 1971, A\&A, 11, 218

Thomas, G. E. 1978, Ann. Rev. Earth Planet. Sci., 6, 1978

Witte, M. 2004, A\&A, 426, 835

Wood, B. E., Izmodenov, V. V., Linsky, J. L., \& Malama, Yury, G. 2007, ApJ, 657,609

Wood, B. E., Izmodenov, V. V., Linsky, J. L., \& Alexashov, D. 2007, ApJ, 659, 1784

Woods, T. N., \& Eparvier, F. G., McClintock, W., et al. 2005, J. Geophys. Res., 110, A01312

Zank, G. P., \& Mueller, H.-R. 2003, J. Geophys. Res., 108, 1240 